\title{
Preterm birth and stillbirth rates during the COVID-19 pandemic: a population-based cohort study
}

\author{
Prakesh S. Shah MD, Xiang Y. Ye MSc, Jie Yang PhD, Michael A. Campitelli MPH
}

Cite as: CMAJ 2021 August 3;193:E1164-72. doi: 10.1503/cmaj.210081

\begin{abstract}
BACKGROUND: Conflicting reports have emerged for rates of preterm births and stillbirths during the COVID-19 pandemic. Most of these reports did not account for natural variation in these rates. We aimed to evaluate variations in preterm birth and stillbirth rates before and during the COVID-19 pandemic in Ontario, Canada.
\end{abstract}

METHODS: We conducted a retrospective cohort study using linked population health administrative databases of pregnant people giving birth in any hospital in Ontario between July 2002 and December 2020. We calculated preterm birth and stillbirth rates. We assessed preterm birth at 22-28, 29-32 and 33-36 weeks' gestation, and stillbirths at term and preterm gestation. We used Laney control $\mathrm{P}^{\prime}$ charts for the 18-year study period (6-mo observation periods) and interrupted time-series analyses for monthly rates for the most recent 4 years.

RESULTS: We evaluated 2465387 pregnancies, including 13781 that resulted in stillbirth. The mean preterm birth rate for our cohort was $7.96 \%$ (range $7.32 \%$ $8.59 \%)$. From January to December 2020, we determined that the preterm birth rate in Ontario was $7.87 \%$, with no special cause variation. The mean stillbirth rate for the cohort was $0.56 \%$ (range $0.48 \%-0.70 \%$ ). From January to December 2020, the stillbirth rate was $0.53 \%$, with no special cause variation. We did not find any special cause variation for preterm birth or stillbirth subgroups. We found no changes in slope or gap between prepandemic and pandemic periods using interrupted timeseries analyses.

INTERPRETATION: In Ontario, Canada, we found no special cause variation (unusual change) in preterm birth or stillbirth rates, overall or by subgroups, during the first 12 months of the COVID19 pandemic compared with the previous 17.5 years.

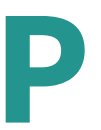

reterm birth (birth before 37 weeks' gestation) is a leading cause of mortality and morbidities in the neonatal period, ${ }^{1}$ childhood and adulthood. ${ }^{2}$ Stillbirth has devastating consequences for families. ${ }^{3}$ The causes of both preterm birth and stillbirth are multifactorial. During the pandemic, reports described reductions in preterm birth rates in Denmark, ${ }^{4}$ the Netherlands, ${ }^{5}$ Ireland ${ }^{6}$ and the United States. ${ }^{7}$ At the same time, increases in stillbirth rates were reported from the United Kingdom, ${ }^{8}$ Italy, ${ }^{9} \mathrm{Nepal}^{10}$ and India, ${ }^{11}$ with or without changes in rates of preterm births. Meta-analyses have emerged with differing conclusions. ${ }^{12,13}$ Some speculated reasons for reductions in preterm births included reductions in physical activity during pregnancy, reduced stress related to work-life balance, less exposure to infection, fewer medical interventions, reduced travel and pollution, ${ }^{14}$ and improved hygiene and rest. Proposed reasons for increases in preterm birth rates include higher stress due to worry about the pandemic, employment or financial challenges, home schooling and reduced maternity services. ${ }^{15}$ Less stringent fetal surveillance from reduced attendance at medical appointments for fear of infection, cancellation of face-to-face appointments and reduced staffing for maternity services are possible reasons for increased rates of stillbirths. Thus, it is important to evaluate preterm births and stillbirths simultaneously to understand the true impact. ${ }^{16}$

Some previous reports compared preterm birth and stillbirth rates during the pandemic to similar time periods in the past few years. However, within a jurisdiction, these rates are known to fluctuate between epochs ${ }^{17}$ and, thus, it is preferable to evaluate rates over longer periods to establish whether observed variations are usual (common cause variation) or unusual (special cause variation). Our objective was to evaluate whether the COVID-19 pandemic affected preterm birth or stillbirth rates in Ontario by comparing rates for the early COVID-19 pandemic time period with rates from the previous 17.5 years to identify patterns of variation. 


\section{Methods}

\section{Study design}

We conducted a population-based, retrospective cohort study using data from an administrative database in the province of Ontario, Canada, from July 2002 to December 2020.

\section{Population and setting}

We included resident pregnant people aged 13-59 years who delivered in any hospital in Ontario and matched them to their live or stillborn offspring. Ontario contributes about $130000-$ 140000 births per year out of a total about 350000 births in Canada. Facility-based care is covered under the provincial health insurance plan for all residents.

\section{Data sources}

The Canadian Institute for Health Information's Discharge Abstract Database (CIHI-DAD) contains clinical and administrative data for all inpatient admissions in Ontario, including records for pregnant people who deliver in hospital and their newborns. Hospital births accounted for about $98 \%$ of all births ${ }^{18}$ in the province. The Ontario Mother-Baby data set deterministically links maternal hospital delivery and infant birth records using a unique maternal-newborn chart number, with linkage rates exceeding $98 \% .{ }^{18}$

We divided the data records from July 2002 and December 2020 into 6-month observation periods. The first case of SARSCoV-2 infection was recorded in Ontario on Jan. 25, 2020, and strict lockdown measures in Canada were implemented as of Mar. 18, 2020. Since that time, Canada has had a Lockdown Stringency index ${ }^{19}$ of 60-75 on a scale of 0-100 (where 100 represents the strictest lockdown). We designated January to December 2020 as the "pandemic period" because SARS-CoV-2 and COVID-19 were mentioned in the Canadian media from Nov. 17, 2019, with the first recorded case in Wuhan, China. We considered the preceding 35 periods (each of $6 \mathrm{mo}$ ) "free of pandemic effects," and these were used to derive baseline variability. We calculated the rates of preterm birth and stillbirth using data from each public health unit on the number of births among pregnant people living in the region, which included gestational age. To understand differences in outcomes across different settings, we assessed pregnant people living in rural versus urban areas and according to their neighbourhood income quintile. ${ }^{20}$ We defined a rural region as jurisdictions outside the commuting zone of centres with populations of 10000 or more. ${ }^{21}$ We used data from the Statistics Canada Postal Code Conversion file for neighbourhood income quintiles, which divides areas into quintiles based on neighbourhood income, with quintile 1 representing lowest area-level income and quintile 5 representing highest area-level income. SARS-COV-2 positivity rates during the pandemic were higher in 4 of the 34 public health units of Ontario, and we compared outcome rates in these locations to the rest of the province. The 4 public health units were Toronto (population 2731571), York Region (population 1109 909), Peel Region (population 1381744) and Ottawa (population 934243). We merged the remaining public health units into an "other" region (population 8412533).

\section{Outcomes}

\section{Preterm birth}

We classified live births as those occuring between 21 weeks 0 days' gestation and 36 weeks 6 days' gestation as preterm births. Although the limit of viability has changed during the years that we reviewed in our study, we used this definition to keep the calculation of the rate of preterm birth consistent. However, we excluded 717 infants recorded as births at 21 weeks' gestation from the subgroup analyses. We planned to look at subgroups of preterm births at 22-28, 29-32 and 33-36 weeks' gestation.

\section{Stillbirth}

We defined stillbirth as fetal death before the complete expulsion or extraction of products of conception after at least 20 weeks of pregnancy. ${ }^{22}$ We used International Statistical Classification of Diseases and Related Health Problems, 10th Revision (ICD-10) codes Z37.1, Z37.3, Z37.4, Z37.6 and Z37.7 to identify stillbirths. We also evaluated term stillbirths (at $\geq 37$ weeks 0 days' gestation) and preterm stillbirths (at $<37$ weeks' gestation). The data for stillbirths were derived from maternal delivery records and represent a pregnancy-level denominator.

\section{Statistical analysis}

We used 2 approaches for data analysis (details in Appendix 1A, available at www.cmaj.ca/lookup/doi/10.1503/cmaj.210081/ tab-related-content).

\section{Identification of special cause variation}

We estimated the rates of preterm birth and stillbirth for each of the 6-month periods from July-December 2002 to JulyDecember 2020. Because the sample sizes were large, we used Laney control $\mathrm{P}^{\prime}$ charts $^{23}$ to describe and detect common cause (usual) and special cause (unusual) variations by plotting crude rates, the upper control limit (UCL) and lower control limit ( $(\mathrm{CL})$. We chose 6 months as the time period because control chart analysis requires at least 24 data points to identify special cause variation. Because we had data for 18.5 years, including 1 year of the pandemic period, this allowed us to assess variation using 37 data points. We identified special cause variation using standard definitions. ${ }^{24,25}$ During the entire study period, if we identified special cause variation, we calculated a new mean, UCL and LCL from the start point of the special cause variation and adjusted the chart. However, for the pandemic period (JanuaryDecember 2020), we used only 1 rule of point estimate outside of the control limit to detect special cause variation.

\section{Identification of change in slope}

To examine recent change, we estimated the rates of preterm birth and stillbirth for each month of the 4 latest study years (January 2017 to December 2020). We chose a 1-month interval to study change in trend in relation to change during the pandemic period to ensure that we had at least 8 data points during the pandemic period and that the number of events in the individual cell exceeded the threshold for data release. The 4-year 
period provided us with 48 data points for analyzing the change in slope. We used the interrupted time-series analysis method ${ }^{26}$ to evaluate if there was a sudden change at the cut-off for the start of the pandemic period (December 2019) and if there was a difference in the rates of change of preterm birth and stillbirth rates over time (slopes) between the pandemic period and the nonpandemic period.

We used SAS version 9.4 (SAS Institute Inc.) and $\mathrm{R}$ version 4.0.1 (www.r-project.org) to perform data management and statistical analyses. We considered a 2 -sided $p$ value of less than 0.05 as statistically significant.

\section{Ethics approval}

The study was approved by the Research Ethics Board at Mount Sinai Hospital, Toronto (approval no. 20-0205-C).

\section{Results}

During the study period from July 2002 to December 2020, we identified 2474284 maternal-newborn records from inpatient facilities. Of these, 8897 records met our exclusion criteria. The remaining 2465387 records of delivery and associated births were included in the analysis of special cause variation (Appendix 1B). Across our study period, the mean number of total births was 66259 (range 62574 to 69 839) per half year. We found that the mean gestational age (GA) at birth was 38.7 (SD 2.0) weeks, and the mean birthweight was 3344 (SD 584) grams.

\section{Preterm birth rate}

The mean preterm birth rate for the entire cohort was $7.96 \%$, ranging from $7.32 \%$ to $8.59 \%$ over study periods. Figure 1 shows the variation of preterm birth rates over the entire study period (raw data in Appendix 1C). We found that there was no special cause variation in preterm birth rate during the 12 months of the pandemic period. Our interrupted time-series analysis showed, during the period of January 2017 to December 2020, no significant decrease or increase in the rate of change, trends, or cut-off (gap) between the prepandemic and pandemic periods, despite the visual perception of reduction in slope after January 2020 (Figure 2).

We evaluated preterm birth rates at 22-28 weeks' GA (mean $0.57 \%$ ), at $29-32$ weeks' GA (mean $1.01 \%, 0.92 \%$ and $0.88 \%$; there are 3 rates because of 2 special cause variations) and at 33-36 weeks' GA (mean 6.42\%). The control chart for GA at 29-32 weeks showed special cause variations (decrease) in period 2013 (July-December) and period 2017 (July-December), and we recalibrated charts from those points. We did not identify any special cause variations during the pandemic period compared with previous periods (Figure 3; raw data in Appendix $1 C$ ). Interrupted time-series analysis of the GA subgroups (Appendix 1D) also did not show any significant change in the rates of preterm births between periods in the subgroups, except for GA at 33-36 weeks: the difference between the 2 slopes was almost significant.

\section{Stillbirth rate}

The mean stillbirth rate was $0.56 \%$ and ranged from $0.48 \%$ to $0.70 \%$ over the study period. We identified special cause variation in the form of reduced stillbirth rate in the July-December 2012 period, with a mean rate of $0.59 \%$ before and $0.52 \%$ after the change. We readjusted the charts from the subsequent period. Figure 4 shows the variations in rates of stillbirths over the study period (raw data in Appendix $1 \mathrm{E}$ ). We found no special cause variation in stillbirth rate for the pandemic period. Our interrupted time-series analysis showed no significant change in slope, trends or cut-off (gap) between the prepandemic and pandemic periods (Figure 5).

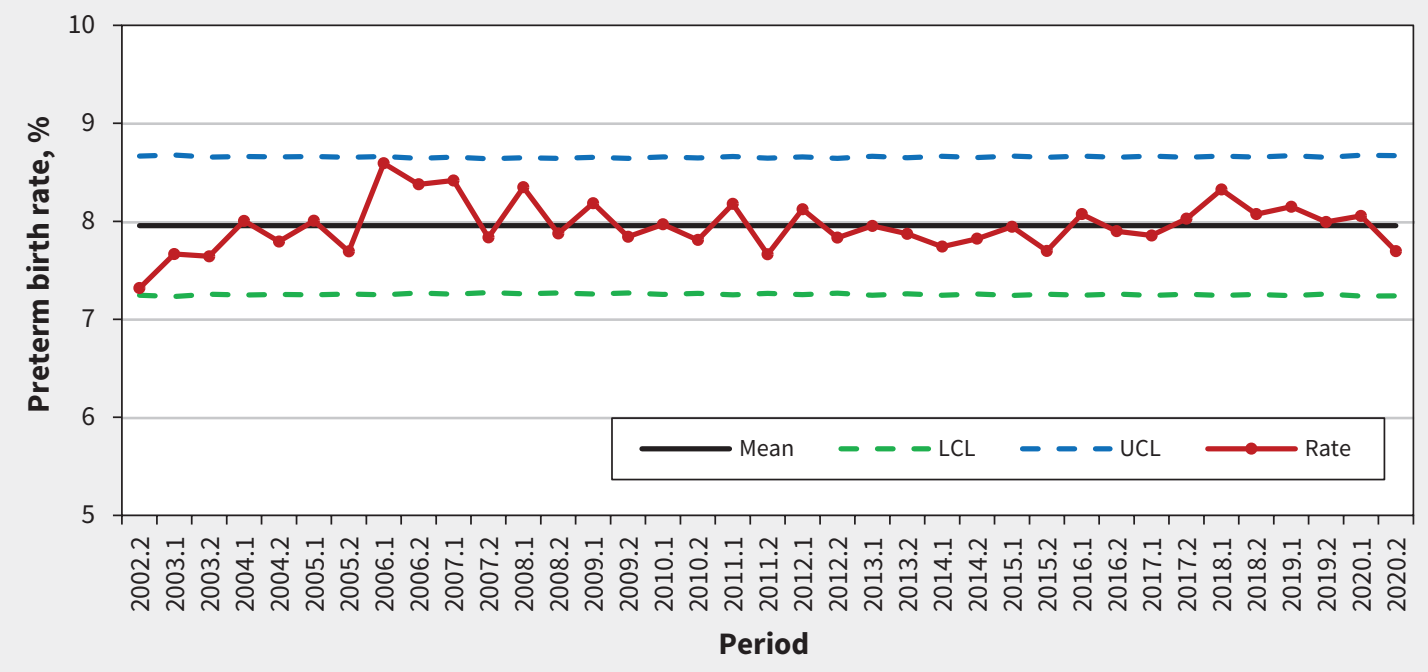

Figure 1: Control chart showing variation in rate of preterm births from July 2002 to December 2020 in 6-month epochs. For example, 2002.2 denotes July to December 2002, and 2003.1 denotes January to June 2003. Note: LCL = lower control limit, UCL = upper control limit. 


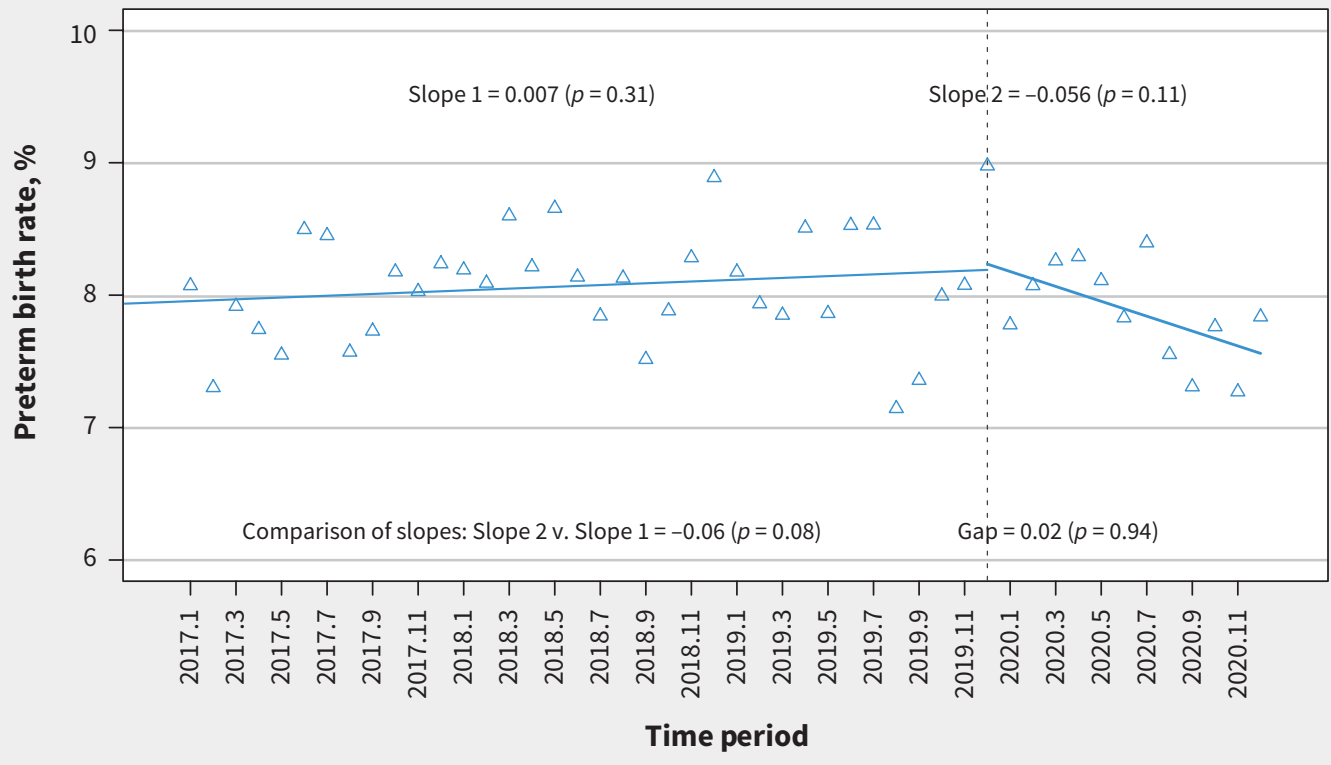

Figure 2: Interrupted time-series analysis of the rate of preterm births, by month, from January 2017 to December 2020. For example, 2017.1 denotes January 2017. Slope 1 and Slope 2 are the slopes of trends in preterm birth rate from January 2017 to December 2019 (nonpandemic period) and from January to December 2020 (pandemic period), respectively. Gap is the change in the rate at the turning point between the 2 periods (nonpandemic $v$. pandemic period). The vertical broken line represents December 2019, which separated the pandemic period from the prepandemic period.

We evaluated stillbirth at term gestation and identified a special cause variation in the form of reduced stillbirth rate in the July-December 2014 period. The mean rate before the change was $0.16 \%$ and after was $0.13 \%$. We readjusted the mean, UCL and $L C L$ from the subsequent period. The mean stillbirth rate at preterm gestation was $0.41 \%$. We did not identify any special cause variation in preterm stillbirth during the pandemic period (Appendix 1E and F). The results of interrupted time-series analysis (Appendix 1G) also did not show any significant changes in any parameters.

\section{Subgroup analysis}

\section{Preterm birth rate}

We found that the mean rate of preterm birth was $7.64 \%$ for mothers in rural regions and $7.99 \%$ for mothers in urban regions (Appendix $1 \mathrm{H}$ ). The control chart did not identify special cause variation in either rural or urban mothers (Appendix 1I). The mean rates of preterm birth by neighbourhood income quintiles 1-5 were $8.39 \%, 8.12 \%, 7.94 \%, 7.67 \%$ and $7.55 \%$, respectively (Appendix 1J). The control chart for income quintile 1 showed special cause variation (an increase) in the July-December 2016 period (with a mean of $8.22 \%$ before and $8.92 \%$ after the period), and we adjusted the charts (Appendix $1 \mathrm{~K}$ ). The control chart did not identify special cause variation in any other quintiles, although the rates of preterm births decreased as neighbourhood income increased. We identified no special cause variation in any income quintile group during the pandemic period (Appendix $1 \mathrm{~K}$ ) and no special cause variation in the 4 regions of interest with high rates of SARS-CoV-2 positivity (Appendix $1 \mathrm{~L}$ and M).

\section{Stillbirth rate}

We found that the mean rate of stillbirths for pregnant people from both rural and urban regions was $0.56 \%$ (Appendix $1 \mathrm{~N}$ ). The control chart for urban stillbirth rates showed special cause variation (a decrease) in the July-December 2012 period, and we adjusted the charts from that point. The control chart did not identify special cause variation for pregnant people in rural or urban regions during the pandemic period (Appendix 10). Mean stillbirth rates by neighbourhood income quintiles $1-5$ were $0.70 \%, 0.59 \%, 0.55 \%, 0.48 \%$ and $0.44 \%$, respectively (Appendix $1 \mathrm{P}$ ). The control chart did not identify special cause variation in any of the quintiles, although the rates of stillbirth decreased as neighbourhood income increased (Appendix 1Q). The control charts for regions of high SARS-CoV-2 positivity showed special cause variation (decreases) in the July-December 2012 period within the Toronto Public Health Unit region, and we adjusted the charts from that point. There was no special cause variation identified in these 4 regions during the pandemic period (Appendix $1 \mathrm{R}$ and S). Data on monthly rates of preterm births and stillbirths during the last 4 years of the study are presented in Appendix $1 T$ and $U$, respectively.

\section{Interpretation}

We identified no special cause variation in rates of preterm births and stillbirths during the first 12 months of the COVID-19 pandemic compared with the previous 17.5 years in Ontario. We identified no special cause variation in subgroups of very preterm, moderately preterm and late preterm births; term 


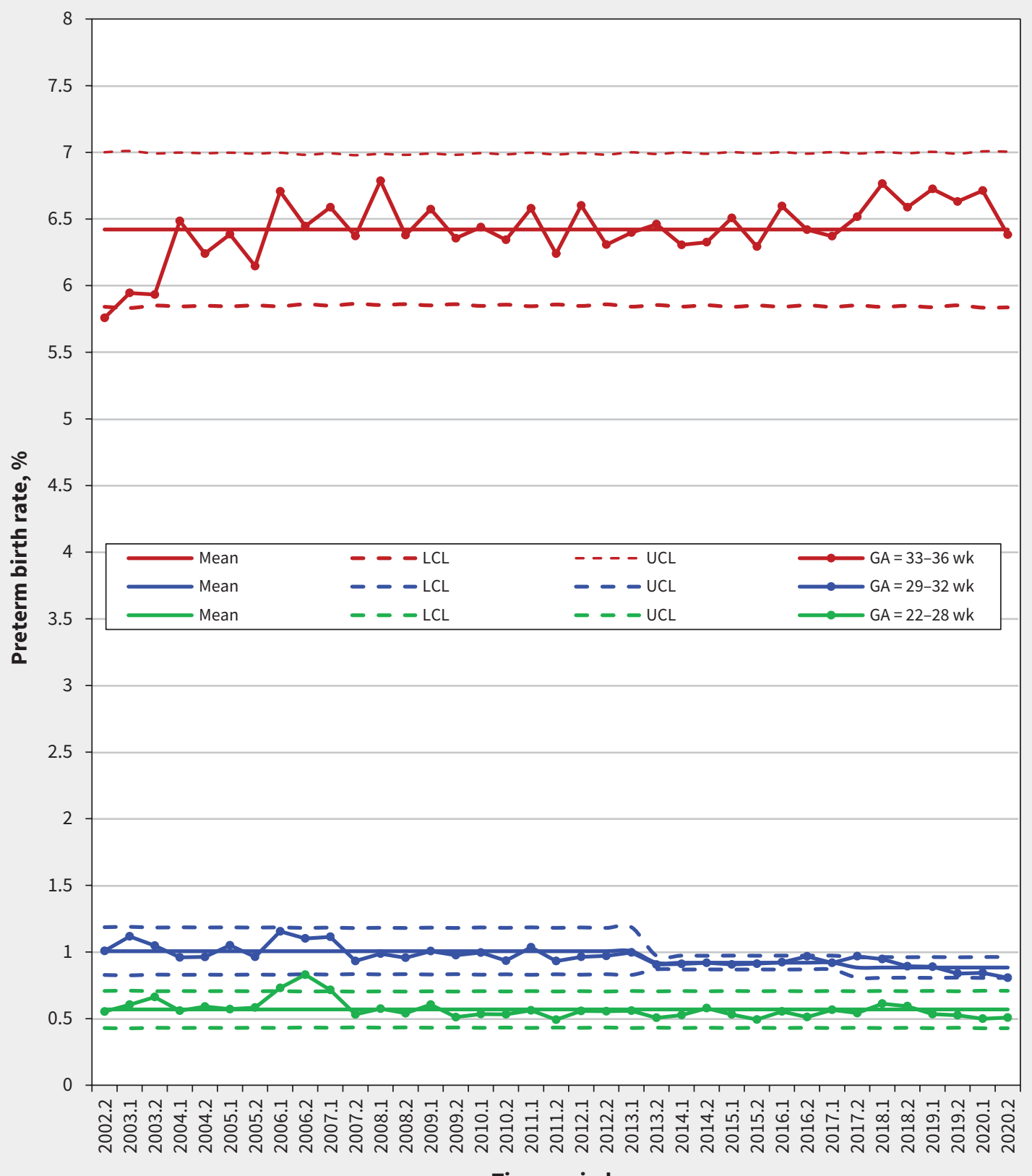

Time period

Figure 3: Control chart showing variation in rate of preterm births from July 2002 to December 2020 in 6-month epochs, by gestational age group. For example, 2002.2 denotes July to December 2002, and 2003.1 denotes January to June 2003. Note: GA = gestational age at birth, LCL = lower control limit, $\mathrm{UCL}=$ upper control limit.

stillbirths; and preterm stillbirths. We found no special cause variations based on the following features of maternal residence location: rural or urban area, neighbourhood income quintile or living in a public health unit with high COVID-19 prevalence. In addition, our interrupted time-series analysis did not identify statistically significant differences in slopes of rates of change for preterm birth and its subgroups or stillbirth and its subgroups. Some of the special cause variations identified involved rates at time points long before the pandemic period and are of interest for further evaluation.
Findings from different jurisdictions have conflicted. One hospital in Ireland ${ }^{6}$ reported that the rate of very low birthweight was reduced to 2.17 per 1000 births $(n=3)$ during January-April 2020 compared with 8.18 per 1000 births ( $n$ varied from 9 to 18/yr) during January 2001 to April 2019. In contrast, another centre in Ireland ${ }^{27}$ reported no differences in perinatal deaths or preterm births during the COVID-19 pandemic period when compared with rates in 2018 and 2019. A nationwide prevalence study involving 31180 singleton live births in Denmark reported significantly lower rates of preterm neonates ( $<28$ weeks' gestation $[n=1]$ during the 


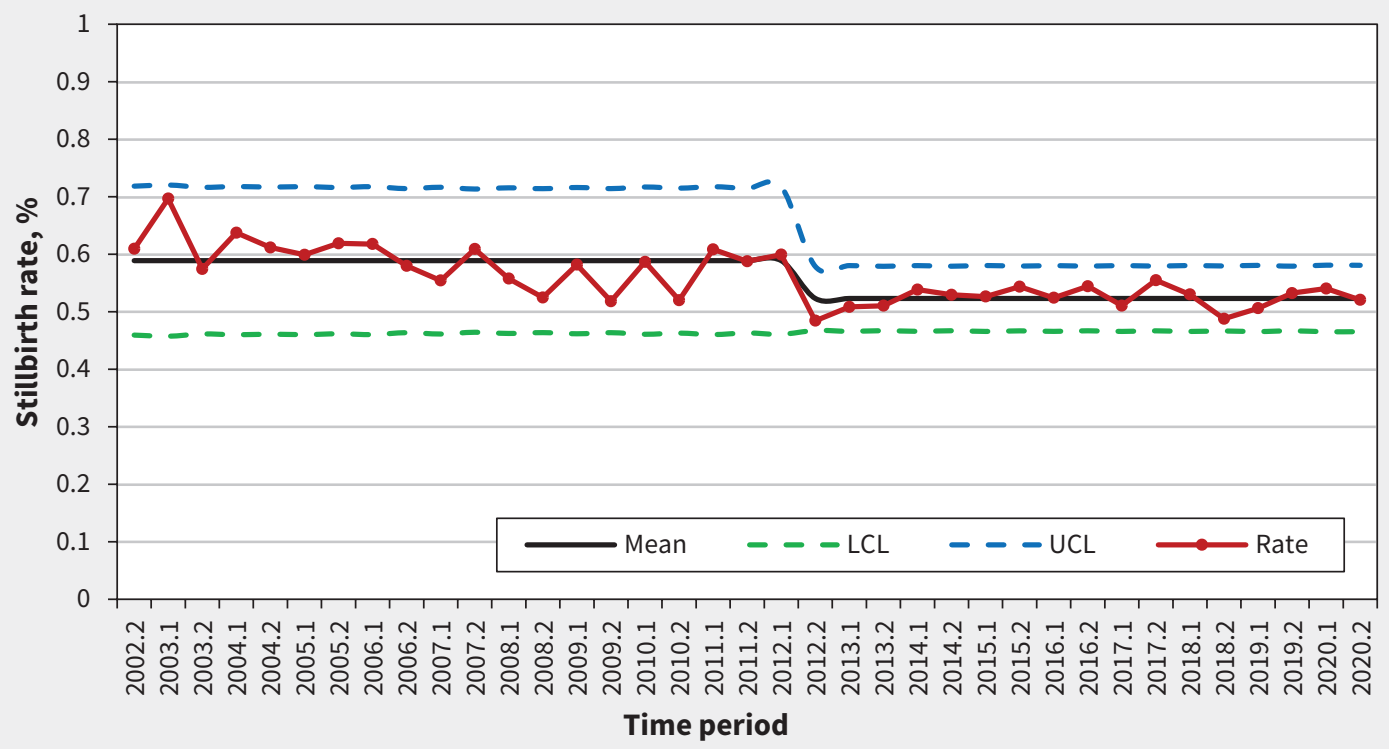

Figure 4: Control chart showing variation in rate of stillbirths from July 2002 to December 2020 in 6-month epochs. For example, 2002.2 denotes July to December 2002, and 2003.1 denotes January to June 2003. We found special cause variation in period 2012.2, with 8 points below the mean; thus, the mean was adjusted from that point onward. Note: $L C L=$ lower control limit, $U C L=$ upper control limit.

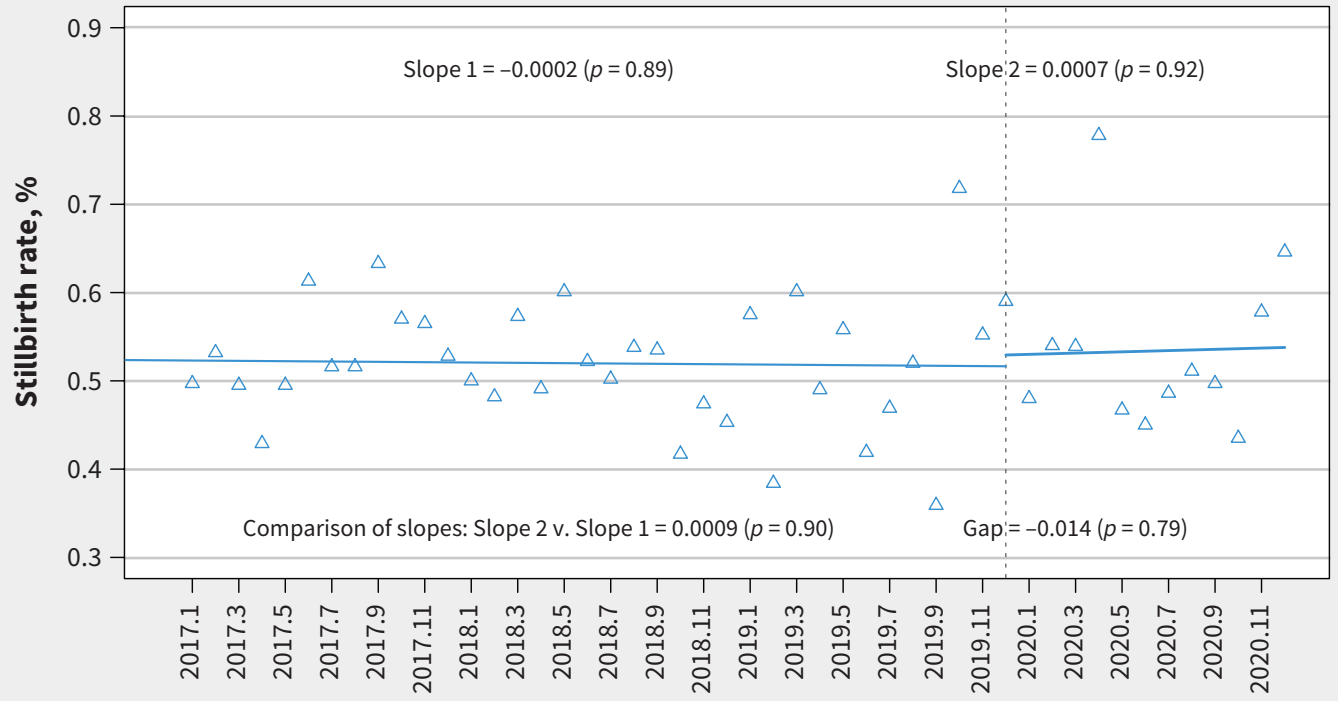

Time Period

Figure 5: Interrupted time-series analysis of the rate of stillbirths, by month, from January 2017 to December 2020. For example, 2017.1 denotes January 2017. Slope 1 and Slope 2 are the slopes of trends in the rate of stillbirths from January 2017 to December 2019 (nonpandemic period) and January to December 2020 (pandemic period), respectively. Gap is the change in the rate at the turning point of the 2 periods (nonpandemic v. pandemic period). The vertical broken line represents December 2019, which separated the pandemic period from the prepandemic period.

lockdown between Mar. 12 and Apr. 14, 2020, compared with rates for 2015-2019 [ $n=57]) .{ }^{4}$ An analysis to investigate the association between the national implementation of COVID-19 mitigation measures and the incidence of preterm birth in the Netherlands reported reductions in preterm birth rate using various time windows surrounding Mar. 9, 2020 (odds ratio [OR] 0.77, 95\% confidence interval $[\mathrm{Cl}] 0.66-0.91$ for the 2 mo before and after Mar. 9, 2020; OR 0.85 , $95 \% \mathrm{Cl} 0.73-0.98$ for the 3 mo before and after 
Mar. 9, 2020; and OR 0.84, 95\% Cl 0.73-0.97 for the 4 mo before and after Mar. 9, 2020). ${ }^{5}$ However, the odds were not statistically significant when Mar. 15 or Mar. 23, 2020, were used as anchors. A study that assessed the impact of the COVID-19 pandemic on preterm birth rates in California reported no difference in rates of preterm birth during April-July 2020 compared with an aggregated rate for the same 4 months over the previous 4 years $(7.41 \%$ v. $7.34 \%$; $p=$ $0.4) .{ }^{28} \mathrm{~A}$ study that compared maternal, obstetrical and neonatal outcomes of singleton pregnancies at 1 centre in Israel reported no differences in rates of preterm births during March 20 to June 27,2020 , compared with the same period in 2019 (6.7\% v. 8.0\%; $p=$ $0.07)$, and the same periods in the preceding 10 years $(6.7 \% \mathrm{v}$. $7.2 \% ; p=0.4) .{ }^{29}$ However, this study also found a significant reduction in preterm births at $<34$ weeks' gestation during the 2020 study period $(1.2 \%, 2.7 \%$ and $2.1 \%$ in the same respective periods). In a 2021 research letter, researchers at a large health system in New York City reported no change in rates of preterm births and stillbirths in their patient population between Mar. 15 and May 15, 2020. ${ }^{30} \mathrm{~A}$ retrospective report from one centre in London, UK, found that the rate of stillbirths (fetal death $\geq 24$ weeks' gestation) was significantly higher during the COVID19 pandemic period (Feb. 1 to June 14, 2020) compared with the prepandemic period (Oct. 1, 2019, to Jan. 31, 2020) (9.31 v. 6.93 per 1000 births, respectively), but no significant differences for rates of preterm birth. ${ }^{8}$ In 2020, researchers in Rome, Italy, evaluated perinatal data from a regional hospital discharge abstract database and reported that compared with the same period in 2019, there was a reduction in late preterm births $(5.93 \%$ v. $4.62 \%$; $p<0.001)$ but a threefold increase in stillbirths $(1.07 \%$ v. 3.23\%; $p=0.002)$ from March to May 2020. ${ }^{9}$ A 2020 prospective observational study in Nepal reported that the stillbirth rate increased to 21 per 1000 births during the lockdown period compared with 14 per 1000 births during prelockdown $(p=0.0002)$, and neonatal mortality increased to 40 per 1000 births compared with 13 per 1000 births $(p=0.002)$, associated with a decline of more than $50 \%$ in institutional births during the lockdown period. ${ }^{10} \mathrm{~A}$ retrospective analysis involving pregnant people from 4 centres in India reported a $43.2 \%$ reduction in rates of hospital admissions for pregnant people with significant increases in maternal mortality $(0.20 \%$ v. $0.13 \% ; p=0.01)$ and intrauterine deaths or stillbirths (3.15\% v. $2.25 \% ; p=0.02$ ) during the lockdown period (Mar. 25 to June 2, 2020) compared with the prelockdown period (Jan. 1 to Mar. 24, 2020). ${ }^{11}$ A 2021 study that evaluated whether rates of preterm birth, spontaneous preterm birth, medically indicated preterm birth and stillbirth changed during the COVID-19 pandemic compared with prepandemic rates using data from the Geobirth pregnancy cohort of all births in 2 hospitals in Philadelphia reported no change in the incidence of preterm births or stillbirths during the pandemic period (March-June 2020) compared with the same months in 2018 and 2019.

Overall, some studies have reported concerning rises in stillbirths, while others have identified reductions in early preterm births. These were summarized recently in 2 systematic reviews in 2021. A review of 12 studies reported an increase in stillbirths and no change in preterm birth rates. ${ }^{12}$ Another review of 28 studies concluded that there was no change in stillbirth rates; however, the authors did find a reduction in the unadjusted estimates of preterm births in single-centre or local studies but not in regional or national studies. ${ }^{13}$ Therefore, findings so far have been inconsistent, with individual studies having variable comparators, highlighting the need for more studies like ours.

Some contributing factors for preterm birth and stillbirth that have been reported in previous studies include infection, inflammation, stress, medical- or pregnancy-induced disorders, genetic predisposition, environmental factors, race/ethnicity and previous obstetric concerns; however, in many instances the cause remains unknown. ${ }^{32}$ Several theories have been suggested about the possible mechanisms.

Some progress has been made toward reducing stillbirth rates over time; however, the progress has been slow. ${ }^{3}$ If the speculations that fewer preterm babies were born during the pandemic ${ }^{33}$ were true, then the pandemic could have provided an opportunity to explore the prevention of preterm birth. However, longitudinal data assessments and results from our study have shown that careful evaluation using appropriate techniques would be required and, when applied, did not show unusual changes in preterm birth or stillbirth rates. The findings of increased stillbirth rates observed in India and Nepal were hypothesized to be due to reduced access to tertiary care facilities; however, the findings of no change in stillbirth rates observed in most other high-income countries ${ }^{27,30,31}$ are similar to ours. Reduced rates of preterm birth that were reported in Denmark, 1 centre in Ireland, Israel and the Netherlands contrast with our findings and other reports; however, none of those studies had information on stillbirth.

Strengths of our study included the geographically defined, population-based cohort; the context of provincially funded health care with relatively few barriers to access to care; the methodologically rigorous evaluation using a valid, multicentre database, and the ability to assess the effects of maternal residence and a proxy measure for socioeconomic status.

\section{Limitations}

Our study was a retrospective evaluation using an administrative linkage database, which is prone to registration errors, although this database has been well-curated and used in multiple projects with a high degree of reliability. The mean number of total births per 6-month period in the prepandemic period was 66425 (range 62574 to 69840 ), while the number of live births in the 2 pandemic periods averaged to about 63370 per 6 months, suggesting a reduced birth rate. This could be due to births to Canadian people living overseas who would usually have returned to Canada for childbirth (but who could not because of travel restrictions), delayed registration by hospitals or increased home births (however, more home births is less likely, as the scope of practice for midwives for home births has not changed during the pandemic). We lacked case-level medical details to discern whether there were differences in the reasons for preterm birth or stillbirth. Our choice of Jan. 1, 2020, as the start date for the pandemic period could be challenged; however, we believe using a lockdown date to define the start of this period could also be criticized, because the timing of the declaration of a pandemic 
and the country's response does not necessarily coincide with onset of pandemic-related stress in its population. News about the pandemic was common starting from the end of 2019. Our discontinuity analyses evaluated monthly change as well.

The effects of pandemic-related mitigation measures and compliance with them could have differential effects in regional and local settings. In some areas and in certain people, the restrictions could have had beneficial effects; in other areas or people, they may have been counterproductive. International efforts are currently underway to understand the global impact of COVID-19 on pregnancy and childbirth. ${ }^{34}$ These studies could provide hints into the associations between population-wide infection, compliance with mitigation measures, and outcomes.

\section{Conclusion}

In a rigorous population-based cohort of hospital-based births in Ontario, Canada, we identified no special cause variation (unusual change) in the incidences of preterm birth or stillbirth or their subgroups during the first year of the COVID19 pandemic period compared with data from the previous 17.5 years.

\section{References}

1. Liu L, Oza S, Hogan D, et al. Global, regional, and national causes of child mortality in 2000-13, with projections to inform post-2015 priorities: an updated systematic analysis. Lancet 2015;385:430-40.

2. Crump C. An overview of adult health outcomes after preterm birth. Early Hum Dev 2020;150:105187.

3. Lawn JE, Gravett MG, Nunes TM, et al. Global report on preterm birth and stillbirth (1 of 7): definitions, description of the burden and opportunities to improve data. BMC Pregnancy Childbirth 2010;10 Suppl 1(Suppl 1):S1. doi: 10.1186/1471-2393-10-S1-S1.

4. Hedermann G, Hedley PL, Bækvad-Hansen M, et al. Danish premature birth rates during the COVID-19 lockdown. Arch Dis Child Fetal Neonatal Ed 2021;106: 3-95.

5. Been JV, Burgos Ochoa L, Bertens LCM, et al. Impact of COVID-19 mitigation measures on the incidence of preterm birth: a national quasi-experimental study. Lancet Public Health 2020;5:e604-11.

6. Philip RK, Purtill H, Reidy E, et al. Unprecedented reduction in births of very low birthweight (VLBW) and extremely low birthweight (ELBW) infants during the COVID-19 lockdown in Ireland: a 'natural experiment' allowing analysis of data from the prior two decades. BMJ Glob Health 2020;5:e003075.

7. Preston E. During coronavirus lockdowns, some doctors wondered: Where are the preemies? The New York Times 2020 July 19. Available: https://www. nytimes.com/2020/07/19/health/coronavirus-premature-birth.html [login required to access content] (accessed $2020 \mathrm{Dec}$. 14).

8. Khalil A, von Dadelszen $\mathrm{P}$, Draycott $\mathrm{T}$, et al. Change in the incidence of stillbirth and preterm delivery during the COVID-19 pandemic. JAMA 2020;324:705-6.

9. De Curtis M, Villani L, Polo A. Increase of stillbirth and decrease of late preterm infants during the COVID-19 pandemic lockdown. Arch Dis Child Fetal Neonatal Ed 2021;106:456

10. Kc A, Gurung R, Kinney MV, et al. Effect of the COVID-19 pandemic response on intrapartum care, stillbirth, and neonatal mortality outcomes in Nepal: a prospective observational study. Lancet Glob Health 2020;8:e1273-81.

11. Kumari V, Mehta K, Choudhary R. COVID-19 outbreak and decreased hospitalisation of pregnant women in labour. Lancet Glob Health 2020;8:e1116-7.

12. Chmielewska B, Barratt I, Townsend R, et al. Effects of the COVID-19 pandemic on maternal and perinatal outcomes: a systematic review and meta-analysis. Lancet Glob Health 2021;9:e759-72.
13. Yang J, D'Souza R, Kharrat A, et al. COVID-19 pandemic and population-level pregnancy and neonatal outcomes: a living systematic review and meta-analysis. Acta Obstet Gynecol Scand 2021 June 6 [Epub ahead of print]. doi: 10.1111/ aogs.14206.

14. Bauwens M, Compernolle S, Stavrakou T, et al. Impact of coronavirus outbreak on $\mathrm{NO}_{2}$ pollution assessed using TROPOMI and OMI observations. Geophys Res Lett 2020 May 8 [Epub ahead of print]. doi: e2020GL087978.

15. Jardine J, Relph S, Magee LA, et al. Maternity services in the UK during the coronavirus disease 2019 pandemic: a national survey of modifications to standard care. BJOG 2021;128:880-9.

16. Homer CSE, Leisher SH, Aggarwal N, et al. Counting stillbirths and COVID 19-there has never been a more urgent time. Lancet Glob Health 2021;9: e10-e11.

17. Chawanpaiboon S, Vogel JP, Moller AB, et al. Global, regional, and national estimates of levels of preterm birth in 2014: a systematic review and modelling analysis. Lancet Glob Health 2019;7:e37-46.

18. Live births and fetal deaths (stillbirths), by place of birth (hospital or non-hospital). Ottawa: Statistics Canada; 2020. Available: https://www150.statcan.gc.ca/t1/tbl1 /en/cv!recreate.action?pid=1310042901\&selectedNodelds=1D7,4D2\&checkedLevels =1D1,1D2,2D1,2D2\&refPeriods=19910101,20180101\&dimensionLayouts=layout2, layout3,layout3,layout2,layout2\&vectorDisplay=false (accessed 2020 Dec. 14).

19. COVID-19: Government Response Stringency Index. Oxford (UK): Our World in Data. Available: https://ourworldindata.org/grapher/covid-stringency-index? tab=chart\&stackMode=absolute\&time=2020-01-22.latest\&country $=\sim$ CAN\&region $=$ World (accessed 2020 Dec. 14).

20. Postal Code ${ }^{\mathrm{OM}}$ Conversion File (PCCF), reference guide 2017. Ottawa: Statistics Canada; 2017. Available: https://www150.statcan.gc.ca/n1/pub/92-154-g/92 -154-g2017001-eng.htm (accessed 2020 Dec. 14).

21. du Plessis V, Beshiri R, Bollman RD, et al. Definitions of rural. Rural and Small Town Canada Analysis Bulletin. Ottawa: Agricultural Division, Statistics Canada; 2001. Cat no 21-006XIE. Available: https://www150.statcan.gc.ca/n1/pub/21 -006-x/21-006-x2001003-eng.pdf (accessed 2020 Dec. 14).

22. Vital Statistics - Stillbirth Database. Deaths 2004. Ottawa: Statistics Canada; 2004. Cat no 84F0211XWE. Available: https://www150.statcan.gc.ca/n1/ pub/84f0211x/2004000/4068009-eng.htm (accessed 2020 Dec. 14).

23. Laney DB. Improved control charts for attributes. Qual Eng 2002;14:531-7.

24. Wheeler DJ. Understanding variation: the key to managing chaos. Knoxville (TN): SPC Press; 1993.

25. Wheeler DJ, Chambers DS. Understanding statistical process control. Knoxville (TN): SPC Press; 1992.

26. Thistlethwaite D, Campbell D. Regression-discontinuity analysis: an alternative to the ex post facto experiment. J Educ Psychol 1960;51:309-17.

27. McDonnell S, McNamee E, Lindow SW, et al. The impact of the COVID-19 pandemic on maternity services: a review of maternal and neonatal outcomes before, during and after the pandemic. Eur J Obstet Gynecol Reprod Biol 2020;255:172-6.

28. Main EK, Chang S-C, Carpenter AM, et al. Singleton preterm birth rates for racial and ethnic groups during the coronavirus disease 2019 pandemic in California. Am J Obstet Gynecol 2021;224:239-41.

29. Meyer R, Bart Y, Tsur A, et al. A marked decrease in preterm deliveries during the coronavirus disease 2019 pandemic. Am J Obstet 2021;224:234-7.

30. Janssen $\mathrm{O}$, Thompson M, Milburn S, et al. The impact of perinatal SARS-CoV2 infection during the peripartum period. Am J Obstet Gynecol MFM 2021;3:100267.

31. Handley SC, Mullin AM, Elovitz MA, et al. Changes in preterm birth phenotypes and stillbirth at 2 Philadelphia hospitals during the SARS-CoV-2 pandemic, March-June 2020. JAMA 2021;325:87-9.

32. Goldenberg RL, Rouse DJ. Prevention of premature birth. N Engl J Med 1998;339:313-20.

33. Fewer babies are being born premature during COVID-19. CTV News 2020 Aug. 8. Available: https://www.ctvnews.ca/health/fewer-babies-are-being -born-premature-during-covid-19-1.5056815 (accessed 2020 Dec. 14).

34. International perinatal outcomes in the pandemic study [iPOP]. Available: https://www.ipopstudy.com/ (accessed 2020 Dec. 14). 
Competing interests: None declared.

This article has been peer reviewed.

Affiliations: Department of Pediatrics and Maternal-Infant Care Research Centre (Shah, Ye, Yang), Mount Sinai Hospital; Department of Pediatrics (Shah), University of Toronto; ICES (Campitelli), Toronto, Ont.

Contributors: Prakesh Shah contributed to the study design, analyzed and intrepreted the data, and drafted the work. Xiang Ye and Jie Yang contributed to the study design, and analyzed and intrepreted the data. Michael Campitelli contributed to the study design, and collected and interpreted the data. All of the authors revised the manuscript, gave final approval of the version to be published and agreed to be accountable for all aspects of the work in ensuring that questions related to its accuracy or integrity are appropriately resolved.

Content licence: This is an Open Access article distributed in accordance with the terms of the Creative Commons Attribution (CC BY-NC-ND 4.0) licence, which permits use, distribution and reproduction in any medium, provided that the original publication is properly cited, the use is noncommercial (i.e., research or educational use), and no modifications or adaptations are made. See: https://creativecommons.org/licenses/ by-nc-nd/4.0/

Funding: This study was supported by the Ontario Health Data Platform, a Province of Ontario initiative to support Ontario's ongoing response to COVID-19 and its related impacts. Prakesh Shah holds an Applied Research Chair in Reproductive and Child Health Services and Policy Research from the Canadian Institutes of Health Research (APR-126340). Prakesh Shah has received funding support from the Canadian Institutes of Health Research for development of the Canadian Preterm Birth Network.
Data sharing: ICES is named as a prescribed entity under Section 45(1) of Ontario's Personal Health Information Protection Act, 2004 (PHIPA). As a requirement of having this status in PHIPA, ICES policies, practices and procedures are reviewed and approved by the Ontario Information and Privacy Commissioner. Access to raw data is governed by confidentiality agreements between ICES and independent investigators as per PHIPA guidelines.

Acknowledgements: The authors thank Dr. Marc Beltempo of McGill University, Montréal, for his suggestions regarding the analyses. They also thank Dr. Heather McDonald Kinkaid, a scientific writer at the Maternal-Infant Care Research Centre (MiCare) at Mount Sinai Hospital, Toronto, for editorial support in the preparation of this manuscript.

Disclaimer: The opinions, results and conclusions reported in this paper are those of the authors and are independent from the funding sources. No endorsement by the Ontario Health Data Platform, its partners or the Province of Ontario is intended or should be inferred. This study contracted ICES Data \& Analytic Services and used de-identified data from the ICES Data Repository, which is managed by ICES with support from its funders and partners: Canada's Strategy for Patient-Oriented Research (SPOR), the Ontario SPOR Support Unit, the Canadian Institutes of Health Research (ClHR) and the Government of Ontario. The opinions, results, and conclusions reported are those of the authors. No endorsement by ICES or any of its funders or partners is intended or should be inferred. Parts of this material are based on data and information compiled and provided by the Canadian Institute for Health Information (CIHI); however, the analyses, conclusions, opinions and statements expressed herein are those of the authors, and not necessarily those of $\mathrm{ClHI}$.

Accepted: June 25, 2021

Correspondence to: Prakesh Shah, Prakeshkumar.Shah@sinaihealth.ca 